\title{
ABC Classification Is Less Useful for Older Koreans Born before 1960
}

Hyungyung Kwon, Sun-Young Lee, Jun Hyun Kim, Sang Pyo Lee, Jeong Hwan Kim, In-Kyung Sung, Hyung Seok Park, and Chan Sup Shim

Department of Internal Medicine, Konkuk University School of Medicine, Seoul, Korea

Background/Aims: In the ABC classification system, group A consists of seronegative subjects without gastric corpus atrophy. This study aimed to determine the prevalence and characteristics of pseudo group A subjects. Methods: Group A subjects were identified among consecutive Korean adults who underwent a serum anti-Helicobacter pylori immunoglobulin G (IgG) test and pepsinogen (PG) assay on the day of endoscopy. Past infection was defined as the presence of either eradication history or endoscopic findings suggesting past infection (i.e., gastric xanthoma, metaplastic gastritis, or advanced atrophy >closed-type 1). Results: Among 2,620 group A subjects, 448 (17.1\%) had eradication history, and 133 (5.1\%) showed endoscopic findings suggesting past infection. Older age (odds ratio [OR], 1.148; 95\% confidence interval [Cl], 1.067 to 1.236) and earlier year of birth (OR, 1.086; 95\% $\mathrm{Cl}, 1.009$ to 1.168) were independent risk factors for classification into pseudo group A, with cutoff points at 50.5 years and birth year of 1959.5, respectively. Positive $H$. pylori test findings were found in 22 subjects (3.1\%) among the 715 subjects who underwent the urea breath test or Giemsa staining on the same day. Current infection was positively correlated with PG I and PG Il levels $(p<0.001)$ but not with age, anti-H. pylori lgG titer, or classification into pseudo group A. Conclusions: Among the group A subjects, $22.2 \%$ had past infection. The risk was higher in subjects older than 50 years, especially those born before 1960. Furthermore, current infection was found in $3.1 \%$ of the subjects and was correlated with increased gastric secretory ability. (Gut Liver 2019;13:522-530 )

Key Words: Age; Helicobacter pylori; Serology; Pepsinogen

\section{INTRODUCTION}

Helicobacter pylori infection and gastric atrophy are well- known causes of gastric cancer. ${ }^{1,2}$ Despite improved eradication and sanitation, $H$. pylori infection is still common in regions where endoscopic gastric cancer screening has been widely performed without eradication. ${ }^{3,4} H$. pylori infection is responsible for 56.5\% of infection-related cancer in Korea, followed by hepatitis B virus (23.9\%), human papillomavirus (11.3\%), and hepatitis C virus (6.0\%). ${ }^{5}$ Although $H$. pylori-seropositivity in urban residents and in younger population is declining in Korea, the prevalence is still high in rural residents and in older population. $^{6}$

Combined serum anti-H. pylori immunoglobulin G (IgG) and pepsinogen (PG) testing is cost-effective for gastric cancer screening in $H$. pylori-seroprevalent population. ${ }^{7,8} \mathrm{ABC}$ classification identifies gastric cancer risk based on the presence of gastric corpus atrophy (PG I level of $\leq 70 \mathrm{ng} / \mathrm{mL}$ and PG I/II ratio of $\leq 3.0$ ) and $H$. pylori-seropositivity. ${ }^{9}$ Group A consists of seronegative subjects without gastric corpus atrophy, group B consists of seropositive subjects without gastric corpus atrophy, group C consists of seropositive subjects with gastric corpus atrophy, and group D consists of seronegative subjects with gastric corpus atrophy. Gastric secretory ability is unimpaired in groups A and B, and gastric cancer risk is lowest in group A. ${ }^{10,11}$ Conversely, gastric secretory ability is impaired in groups $\mathrm{C}$ and $\mathrm{D}$, and gastric cancer risk is highest in group $\mathrm{D}$ owing to previous $H$. pylori infection. ${ }^{10,11}$

Asymptomatic group A subjects are often excluded from the candidates for annual endoscopic gastric cancer screening; however, recent studies show that gastric cancers are not uncommon in group A. ${ }^{12-15}$ This may reflect the misclassification of seroreversed subjects with false negative findings in $\mathrm{ABC}$ classification system. In a recent Japanese study, the risk of gastric cancer in seroreversed subjects after eradication was similar to that in seropositive subjects. ${ }^{12}$ Because there is still a risk of gastric cancer after successful eradication or spontaneous regression, ${ }^{16}$ it is important to distinguish pseudo group A

\footnotetext{
Correspondence to: Sun-Young Lee

Department of Internal Medicine, Konkuk University School of Medicine, 120-1 Neungdong-ro, Gwangjin-gu, Seoul 05030, Korea

Tel: +82-2-2030-7747, Fax: +82-2-2030-7748, E-mail: sunyoung@kuh.ac.kr

Received on September 3, 2018. Revised on November 8, 2018. Accepted on November 22, 2018. Published online May 27, 2019

pISSN 1976-2283 eISSN 2005-1212 https://doi.org/10.5009/gnl18399

(a) This is an Open Access article distributed under the terms of the Creative Commons Attribution Non-Commercial License (http://creativecommons.org/licenses/by-nc/4.0) which permits unrestricted non-commercial use, distribution, and reproduction in any medium, provided the original work is properly cited.
} 
seroreversed subjects among the group A seronegative subjects. In addition to the pseudo group A subjects with past infection, it is important to discriminate group A subjects with current $H$. pylori infection. The aim of this study was to determine the prevalence of the condition and characteristics of pseudo group A among group A subjects.

\section{MATERIALS AND METHODS}

\section{Study subjects}

Korean subjects over 18 years of age who underwent serum anti-H. pylori IgG test and PG assay on the day of upper gastrointestinal endoscopy between January 2010 and June 2016 were analyzed. The subjects were included in the study, if they satisfied the criteria for group A ( $H$. pylori-seronegative subjects without gastric corpus atrophy) as per $\mathrm{ABC}$ classification. ${ }^{9} \mathrm{Ex}-$ clusion criteria were subjects with previous gastrectomy or renal failure. Those who did not answer the questionnaire on past eradication were also excluded. This observational cohort study was approved by the Institutional Review Board of Konkuk University Medical Center (KUH1010626). All subjects provided informed consent before the tests. The study was performed in prospective and retrospective manner by collecting test findings.

\section{Questionnaires and endoscopic examination}

The subjects were asked about their medical history including successful $H$. pylori eradication, gastrectomy, renal failure, hypertension, diabetes mellitus, coronary heart disease, and cerebrovascular attack. Questionnaires on social history included cigarette smoking (never, past, or current) and alcohol drinking (almost none, social, or heavy). Based on the National Institute for Alcohol Abuse and Alcoholism guideline, heavy drinking was defined as $\geq 8$ drinks/wk for women and $\geq 15$ drinks/wk for men. Recent drug intake within last 3 months were asked before the endoscopic examination.

After 12 hours of fasting, endoscopy was performed with the aid of either EG-2990i (Pentax, Tokyo, Japan) or GIF-H260 (Olympus, Tokyo, Japan). Endoscopic findings suggesting past infection were determined by the presence of gastric xanthoma (yellowish plaque), metaplastic gastritis (irregular whitish elevations and/or depressed patchy erythema), or advanced atrophy as described. ${ }^{17,18}$ Advanced atrophy was defined as visible submucosal vessels extending up to the body (>closed-type 1 in Kimura-Takemoto classification) in this study, because the gastric cancer risk is increased from closed-type $2 .{ }^{19}$ Endoscopic images were reviewed by two gastroenterologists (H.K. and S.Y.L.).

\section{Diagnosis for H. pylori-seronegativity}

Serum anti-H. pylori IgG titer was measured by the Vidas $H$. pylori IgG assay (BioMérieux, Marcy-l'Etoile, France) till 2012, and by the Chorus H. pylori IgG (DIESSE Diagnostica Senese,
Siena, Italy) thereafter. For the Vidas H. pylori IgG assay, seronegativity was defined as test value (TV) of $<0.75$ arbitrary units $(\mathrm{AU}) / \mathrm{mL}$. Seropositivity was defined as TV of $\geq 1.00 \mathrm{AU} /$ $\mathrm{mL}$. For the Chorus $H$. pylori IgG assay, seronegativity was defined as TV of $<8.0 \mathrm{AU} / \mathrm{mL}$, and seropositivity was defined as $\geq 12.0 \mathrm{AU} / \mathrm{mL}$. Sensitivity and specificity of the Vidas assay were 89.7\% and 85.5\%, and those of the Chorus assay were 100\% and 75.4\% in Korean subjects, respectively. ${ }^{20}$

Group A subjects were further classified into four subgroups according to their anti-H. pylori IgG titer. For the Vidas $H$. pylori IgG assay, the lowest first-quartile, second-quartile, thirdquartile, and highest fourth-quartile were defined as the antiH. pylori IgG titer of (1) $\leq 0.2 \mathrm{AU} / \mathrm{mL},(2)>0.2$ and $\leq 0.4 \mathrm{AU} / \mathrm{mL}$, (3) $>0.4$ and $\leq 0.6 \mathrm{U} / \mathrm{mL}$, and (4) $>0.6 \mathrm{AU} / \mathrm{mL}$. For the Chorus $H$. pylori IgG assay, the quartiles were defined as the serology titer of (1) $\leq 5.0 \mathrm{AU} / \mathrm{mL},(2)>5.0$ and $\leq 6.0 \mathrm{AU} / \mathrm{mL},(3)>6.0$ and $\leq 7.0$ $\mathrm{AU} / \mathrm{mL}$, and (4) $>7.0 \mathrm{AU} / \mathrm{mL}$, respectively.

\section{Serum pepsinogen assay and $A B C$ classification}

Serum PG levels were analyzed using the latex enhanced turbidimetric immunoassay (HBi Co., Anyang, Korea). ${ }^{10,21}$ Gastric corpus atrophy was defined as a combination of PG I level of $\leq 70 \mathrm{ng} / \mathrm{mL}$ and PG $\mathrm{I} / \mathrm{II}$ ratio of $\leq 3.0 .^{9}$ According to the $\mathrm{ABC}$ classification system, $H$. pylori (-)/gastric corpus atrophy (-) subjects were classified into group A. Subjects showing $H$. pylori (+)/gastric corpus atrophy (-), H. pylori (+)/gastric corpus atrophy (+), and H. pylori (-)/gastric corpus atrophy (+) were classified into groups B, C, and D, respectively.

\section{Definition of pseudo group $A$}

Pseudo group A owing to past infection was defined as seroreversed subjects after $H$. pylori infection, who showed normal findings on anti-H. pylori IgG and PG testings. If there was a history of successful $H$. pylori eradication, subjects were classified into the pseudo group. Subjects with endoscopic findings suggesting past infection (gastric xanthoma, metaplastic gastritis, or advanced atrophy) were also classified into the pseudo group A. ${ }^{17,22}$ Unintended eradication or spontaneous regression was considered as a cause of seroreversion in the latter pseudo group A. Subjects without definite evidence of past infection were classified into true group A.

Current $H$. pylori infection was defined as positive test findings either in the urea breath test (UBT) or on Giemsa staining on the day of endoscopy. UBT was done using Heliview mass spectrometry (MediChems, Seoul, Korea) as described in our previous study. ${ }^{20} \mathrm{H}$. pylori infection was defined as different ${ }^{13} \mathrm{CO}_{2}$ concentration between the baseline and 30 minutes samples of $>2.4$. For Giemsa staining, gastric biopsied specimens were fixed in 95\% ethanol. Thereafter, the samples were embedded in paraffin blocks, and were sectioned for hematoxylin \& eosin and Giemsa stainings as described. ${ }^{21}$ 


\section{Statistical analysis}

Data were analyzed using PASW statistics version 17.0 (SPSS Inc., Chicago, IL, USA). A p-value of $<0.05$ was considered statistically significant. The $\kappa$-value was calculated to evaluate interobserver variability in the endoscopic findings. Differences between the two groups (i.e., true A and pseudo A) were analyzed using the t-test and chi-square test for continuous and categorical variables, respectively. Differences between three or four groups were analyzed by using the post-hoc analysis for continuous variables, and chi-square test with Bonferroni correction for categorical variables. Continuous variables were presented as mean \pm standard deviation, and were presented as proportion (\%). Logistic regression analysis was done to verify independent risk factors for being pseudo group A (being misclassified into group A despite past infection). The values were presented as odds ratio and 95\% confidence intervals. Receiver operating characteristic (ROC) curve analysis was done for significant continuous variables to determine cutoff values for being pseudo group A. Area under the curve and standard error were provided with sensitivity and specificity for each cutoff value.

\section{RESULTS}

\section{Proportion of group A subjects and incidence of gastric neoplasm}

Among 7,178 Korean adults who underwent serum PG assay and the anti-H. pylori IgG test on the same day of endoscopy, 274 were excluded owing to equivocal anti-H. pylori IgG test findings (Fig. 1). The subjects were classified into group
A $(n=2,620)$, group B $(n=3,316)$, group $C(n=898)$, or group D $(\mathrm{n}=70)$. The incidence of gastric adenoma and adenocarcinoma was $0.11 \%$ in group A, 0.33\% in group B, 1.56\% in group C, and 4.29\% in group D (Supplementary Table 1). Among the group A subjects with gastric neoplasm, two were classified into pseudo group A. One subject showed advanced atrophy up to the lower body, and the other showed metaplastic gastritis on the antrum and body.

\section{Proportion of pseudo group A subjects with past $\boldsymbol{H}$. pylori infection}

Among the 2,620 group A subjects, 448 (17.1\%) were classified into the pseudo group owing to previous $H$. pylori eradication (Fig. 1). Additionally, 133 (5.1\%) were also classified into the pseudo group owing to endoscopic findings suggesting past infection. Two gastroenterologists agreed that 139 subjects had endoscopic findings suggesting past $H$. pylori infection (either gastric xanthoma, metaplastic gastritis, or advanced atrophy); a total of 2,434 subjects had no such findings. Discrepancy between the two gastroenterologists was found in 47 subjects $(1.8 \%)$. The $\kappa$-value was $0.846(\mathrm{p}<0.001)$.

\section{True group A versus pseudo group A with past infection}

The true group A subjects were younger $(\mathrm{p}<0.001)$ and had a lower predominance of male sex $(p=0.001)$ than the pseudo group A subjects (Table 1). The true group A subjects were born more recently (mean year of birth: $1962.7 \pm 11.5$ ) than the pseu-

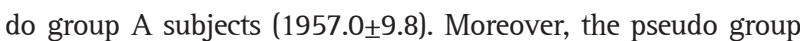
A subjects showed higher prevalence of hypertension $(p=0.002)$, diabetes mellitus ( $p=0.017)$, aspirin use $(p=0.037)$, and cigarette smoking $(\mathrm{p}<0.001)$ than the true group A subjects. The true

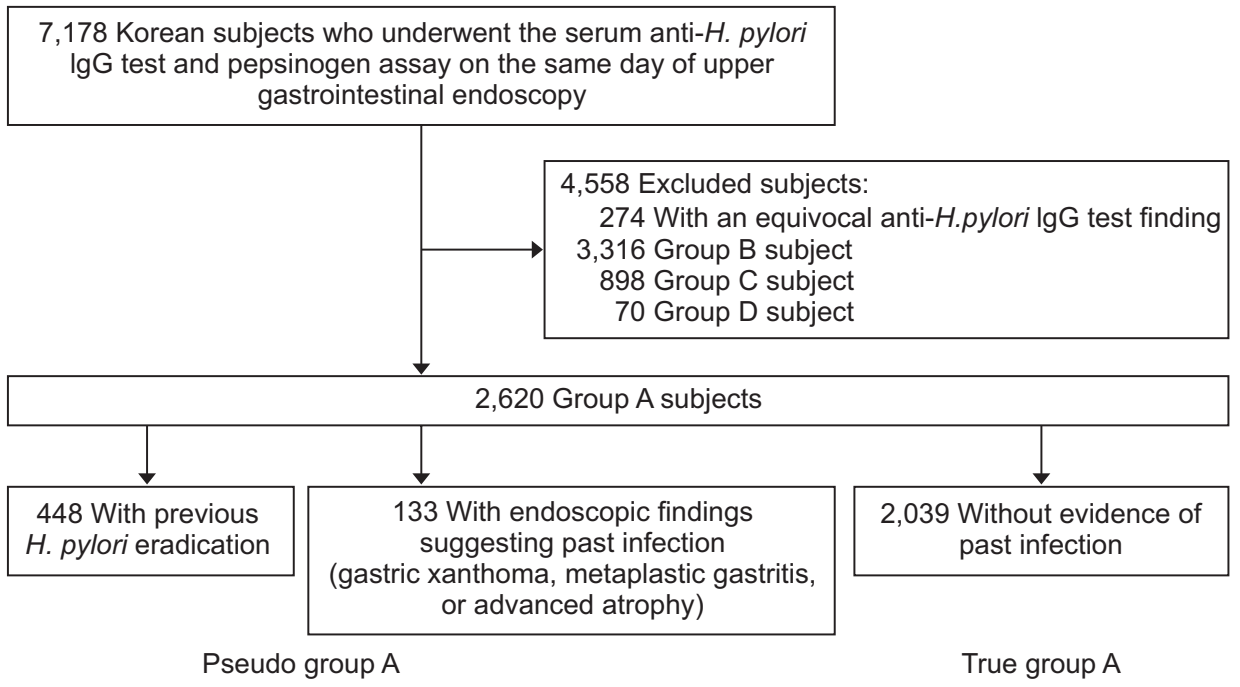

Fig. 1. Flow diagram of study showing subject recruitment and selection criteria. Among the 7,178 Korean subjects, 2,620 Helicobacter pyloriseronegative subjects without gastric corpus atrophy were included in this study. Subjects with eradication history and those with abnormal endoscopic findings suggesting past infection were classified into pseudo group A. Endoscopic findings suggesting past infection were determined by the presence of gastric xanthoma, metaplastic gastritis, or advanced atrophy extending up to the body, because the gastric cancer risk is increased from closed-type $2 .^{19}$ 
Table 1. Baseline Characteristics of the 2,620 Group A Subjects

\begin{tabular}{|c|c|c|c|c|c|c|}
\hline Variable & $\begin{array}{c}\text { True A } \\
(\mathrm{n}=2,039)\end{array}$ & $\begin{array}{l}\text { Pseudo A with } \\
\text { past infection } \\
\qquad(\mathrm{n}=581)\end{array}$ & $\mathrm{p}$-value & $\begin{array}{l}\text { Pseudo A with } \\
\text { eradication history } \\
\qquad(n=448)\end{array}$ & $\begin{array}{c}\text { Pseudo A with } \\
\text { endoscopic } \\
\text { findings suggesting } \\
\text { past infection }(n=133)\end{array}$ & p-value \\
\hline Age, yr & $49.5 \pm 11.3$ & $55.7 \pm 9.7$ & $<0.001$ & $55.1 \pm 9.7$ & $57.5 \pm 9.7$ & 0.016 \\
\hline Year of birth & $1962.7 \pm 11.5$ & $1957.0 \pm 9.8$ & $<0.001$ & $1957.6 \pm 9.8$ & $1954.9 \pm 9.6$ & 0.006 \\
\hline Male sex & $1,061(52.0)$ & $349(60.1)$ & 0.001 & $258(57.6)$ & $91(68.4)$ & 0.025 \\
\hline Hypertension & 365 (17.9) & $128(22.0)$ & 0.002 & $102(22.8)$ & $26(19.5)$ & 0.217 \\
\hline Diabetes mellitus & $129(6.3)$ & $50(8.6)$ & 0.017 & $37(8.3)$ & $13(9.8)$ & 0.770 \\
\hline Coronary heart disease & $67(3.3)$ & $21(3.6)$ & 0.498 & $18(4.0)$ & $3(2.3)$ & 0.273 \\
\hline Cerebrovascular attack & $12(0.6)$ & $2(0.3)$ & 0.747 & $2(0.4)$ & 0 & 0.572 \\
\hline Aspirin & $129(6.3)$ & $48(8.3)$ & 0.037 & $34(7.6)$ & $14(10.6)$ & 0.410 \\
\hline Antithrombotics & $16(0.8)$ & $6(1.0)$ & 0.436 & $6(1.3)$ & 0 & 0.344 \\
\hline Nonsteroidal anti-inflammatory drug & $121(5.9)$ & $33(5.7)$ & 0.887 & $26(5.8)$ & $7(5.3)$ & 0.666 \\
\hline Smoking & & & $<0.001$ & & & 0.075 \\
\hline Current & $332(16.3)$ & $86(14.8)$ & & $57(12.7)$ & $29(21.8)$ & \\
\hline Past & $371(18.2)$ & $137(23.6)$ & & 105 (23.5) & $32(24.1)$ & \\
\hline Never & 801 (39.3) & $176(30.3)$ & & $139(31.0)$ & $37(27.8)$ & \\
\hline No comment & $535(26.2)$ & $182(31.3)$ & & $147(32.8)$ & $35(26.3)$ & \\
\hline Drinking & & & 0.546 & & & 0.594 \\
\hline Heavy* & $96(4.7)$ & $32(5.5)$ & & $24(5.3)$ & $8(6.0)$ & \\
\hline Social & $1,111(54.5)$ & 299 (51.5) & & 222 (49.6) & 77 (57.9) & \\
\hline Almost none & $458(22.5)$ & $119(20.5)$ & & $94(21.0)$ & 25 (18.8) & \\
\hline No comment & 374 (18.3) & $131(22.5)$ & & $108(24.1)$ & 23 (17.3) & \\
\hline
\end{tabular}

Data are presented as the mean \pm SD or number $(\%)$.

*Heavy drinking indicates $\geq 15$ drinks/wk for men and $\geq 8$ drinks/wk for women based on the National Institute for Alcohol Abuse and Alcoholism guideline.

Table 2. Test Findings of the Pseudo Group A Subjects with Past Infection

\begin{tabular}{|c|c|c|c|}
\hline Variable & True A $(n=2,039)$ & $\begin{array}{l}\text { Pseudo A with eradication } \\
\text { history }(\mathrm{n}=448)\end{array}$ & $\begin{array}{l}\text { Pseudo A with endoscopic findings } \\
\text { suggesting past infection }(n=133)\end{array}$ \\
\hline Pepsinogen I, ng/mL & $51.8 \pm 21.1$ & $54.2 \pm 25.4^{*}$ & $51.3 \pm 23.5$ \\
\hline Pepsinogen II, ng/mL & $8.9 \pm 4.5$ & $9.7 \pm 6.2^{*}$ & $9.3 \pm 5.0$ \\
\hline Pepsinogen I/II ratio & $6.4 \pm 4.5$ & $6.2 \pm 3.7$ & $5.7 \pm 1.5^{*}$ \\
\hline Serology assay (Vidas) & $1,160(56.9)$ & $208(46.4)^{*}$ & $73(54.9)^{\dagger}$ \\
\hline \multicolumn{4}{|l|}{ Anti-H. pylori IgG titer } \\
\hline Vidas assay, AU/mL & $0.42 \pm 0.20$ & $0.50 \pm 0.21^{*}$ & $0.44 \pm 0.23^{\dagger}$ \\
\hline Chorus assay, $\mathrm{AU} / \mathrm{mL}$ & $5.72 \pm 1.15$ & $6.28 \pm 1.01^{*}$ & $6.07 \pm 1.28^{\dagger}$ \\
\hline \multicolumn{4}{|l|}{ Serology titer in quartiles } \\
\hline First (lowest) & $578(28.4)$ & $70(15.6)^{*}$ & $36(27.1)^{\dagger}$ \\
\hline Second & $662(32.5)$ & $111(24.8)^{*}$ & $38(28.6)^{\dagger}$ \\
\hline Third & $449(22.0)$ & $129(28.8)^{*}$ & $27(20.3)^{\dagger}$ \\
\hline Fourth (highest) & $350(17.1)$ & $138(30.8)^{*}$ & $32(24.0)^{*,+}$ \\
\hline
\end{tabular}

Data are presented as the mean \pm SD or number $(\%)$.

H. pylori, Helicobacter pylori; IgG, immunoglobulin G.

*Significantly different $(\mathrm{p}<0.05)$ from the true group A subjects; ${ }^{\dagger}$ Significant difference $(\mathrm{p}<0.05)$ between the two pseudo A groups. 
group A subjects showed lower PG I and PG II levels (51.8 \pm 21.1 $\mathrm{ng} / \mathrm{mL}$ and $8.9 \pm 4.5 \mathrm{ng} / \mathrm{mL}$ ) than the pseudo group A subjects $(53.6 \pm 25.0 \mathrm{ng} / \mathrm{mL}$ and $9.6 \pm 5.9 \mathrm{ng} / \mathrm{mL})$. The serum anti-H. pylori IgG titers were also lower in the true group A than their counterparts (Table 2).

\section{Comparisons between the two pseudo group A subjects with past infection}

The pseudo group A subjects with endoscopic findings suggesting past infection were older $(\mathrm{p}<0.001)$ and had a higher predominance of male sex $(\mathrm{p}<0.001)$ than the pseudo group A subjects with eradication history. The pseudo group A subjects with eradication history were born more recently (mean year of birth: 1957.6 \pm 9.8 ) than the pseudo group A subjects with

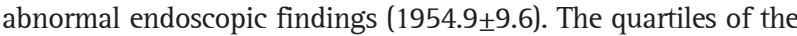

anti-H. pylori IgG titers also differed among the groups (Table 2). The pseudo group A subjects with eradication history showed highest anti-H. pylori IgG titers and PG II levels among all groups.

\section{Differences according to the quartiles of the serum anti- H. pylori lgG titer}

The subjects in the highest fourth quartile were older $(\mathrm{p}=0.001)$ and born earlier $(p<0.001)$ than the subjects in the other quartiles (Table 3). Moreover, these subjects showed the highest mean PG II titer and the lowest PG I/II ratio among the subjects in all four groups. No difference was found among the groups with regard to smoking history $(\mathrm{p}=0.538)$, drinking history $(\mathrm{p}=0.634)$, recent intake of aspirin $(\mathrm{p}=0.378)$, antithrombotic drug intake $(\mathrm{p}=0.995)$, nonsteroidal anti-inflammatory drug

Table 3. Differences Based on Quartiles of the Serum Anti-Helicobacter pylori IgG Titer

\begin{tabular}{|c|c|c|c|c|}
\hline Variable & $\begin{array}{l}\text { First quartile } \\
\quad(\mathrm{n}=684)\end{array}$ & $\begin{array}{l}\text { Second quartile } \\
\quad(\mathrm{n}=811)\end{array}$ & $\begin{array}{c}\text { Third quartile } \\
\quad(\mathrm{n}=605)\end{array}$ & $\begin{array}{c}\text { Fourth quartile } \\
\quad(\mathrm{n}=520)\end{array}$ \\
\hline Age, yr & $50.4 \pm 11.8$ & $50.1 \pm 11.6^{*}$ & $51.2 \pm 10.9^{*,{ }^{\dagger}}$ & $52.5 \pm 10.2^{\dagger}$ \\
\hline Year of birth & $1962.8 \pm 3.3$ & $1961.9 \pm 11.6^{*,+}$ & $1961.0 \pm 11.2^{*, \dagger}$ & $1959.4 \pm 10.5^{\dagger}$ \\
\hline Male sex & $359(52.5)$ & $419(51.7)^{*}$ & $324(53.6)^{*}$ & $308(59.2)^{\dagger}$ \\
\hline Pepsinogen I, ng/mL & $51.7 \pm 22.7$ & $52.4 \pm 22.0$ & $52.2 \pm 21.8$ & $52.5 \pm 21.6$ \\
\hline Pepsinogen II, ng/mL & $8.6 \pm 4.8$ & $9.1 \pm 4.7^{*, \dagger}$ & $9.1 \pm 4.9^{*,+}$ & $9.5 \pm 4.9^{\dagger}$ \\
\hline Pepsinogen I/II ratio & $6.6 \pm 3.2$ & $6.2 \pm 2.9^{*, \dagger}$ & $6.2 \pm 3.1^{*,+}$ & $5.9 \pm 2.3^{\dagger}$ \\
\hline
\end{tabular}

Data are presented as the mean \pm SD or number $(\%)$.

IgG, immunoglobulin G.

${ }^{*}$ Significantly different $(\mathrm{p}<0.05)$ from the subjects in the highest (fourth) quartile; ${ }^{\dagger}$ Significantly different $(\mathrm{p}<0.05)$ from the subjects in the lowest (first) quartile.

Table 4. Comparisons between Helicobacter pylori-Infected Subjects and Noninfected Subjects

\begin{tabular}{|c|c|c|c|}
\hline Variable & $\begin{array}{l}\text { H. pylori infection* } \\
(\mathrm{n}=22)\end{array}$ & $\begin{array}{l}\text { No H. pylori infection } \\
(\mathrm{n}=693)\end{array}$ & p-value \\
\hline Age, yr & $49.6 \pm 13.6$ & $51.9 \pm 11.4$ & 0.352 \\
\hline Year of birth & $1961.7 \pm 13.1$ & $1959.7 \pm 11.3$ & 0.429 \\
\hline Male sex & $15(68.2)$ & $405(58.4)$ & 0.361 \\
\hline Pepsinogen I, ng/mL & $75.3 \pm 36.5$ & $52.0 \pm 22.3$ & $<0.001$ \\
\hline Pepsinogen II, ng/mL & $16.4 \pm 7.9$ & $9.2 \pm 4.2$ & $<0.001$ \\
\hline Pepsinogen I/II ratio & $5.4 \pm 1.7$ & $5.9 \pm 2.3$ & 0.327 \\
\hline Serology assay (Vidas) & $16(72.7)$ & $477(68.8)$ & 0.697 \\
\hline \multicolumn{4}{|l|}{ Serum anti-H. pylori IgG titer, $\mathrm{AU} / \mathrm{mL}$} \\
\hline Vidas assay & $0.54 \pm 0.25$ & $0.43 \pm 0.21$ & 0.052 \\
\hline Chorus assay & $5.95 \pm 0.95$ & $5.96 \pm 0.96$ & 0.984 \\
\hline Quartiles of the serum anti- $H$. pylori IgG, first:second:third:fourth & $4: 3: 7: 8$ & $131: 245: 154: 163$ & 0.155 \\
\hline True group A & $16(72.8)$ & $477(68.9)$ & 0.854 \\
\hline Pseudo group A with eradication history & $3(13.6)$ & $127(18.3)$ & \\
\hline Pseudo group A with endoscopic findings suggesting past infection & $3(13.6)$ & 89 (12.8) & \\
\hline
\end{tabular}

Data are presented as the mean \pm SD or number (\%).

${ }^{*} H$. pylori infection was defined as positive test findings either in the urea breath test or on Giemsa staining on the day of endoscopy. Other variables (i.e., smoking, drinking, drug use, and comorbidities) are not shown owing to the lack of significant difference between groups. 
use $(p=0.136)$, presence of coronary heart disease $(p=0.262)$, cerebrovascular disease $(\mathrm{p}=0.610)$, hypertension $(\mathrm{p}=0.480)$, and diabetes mellitus ( $\mathrm{p}=0.329)$.

\section{Group A subjects with current $\boldsymbol{H}$. pylori infection}

Among the 2,620 included subjects, 715 underwent the UBT or Giemsa staining on the same day of endoscopy (including 130 pseudo group A subjects with eradication history and 92 pseudo group A subjects with endoscopic findings suggesting past infection). Twenty subjects underwent both the UBT and Giemsa staining, and there was no discrepancy between the two tests in these subjects (all negative test findings). Other 599 subjects underwent Giemsa staining only, and 96 underwent UBT only. Positive test findings were found in 22 subjects (3.1\%) (Supplementary Table 2). Among all variables, only the PG I and PG II levels differed between the $H$. pylori test-positive and -negative subjects (Table 4). The $H$. pylori-test findings were not affected by the anti-H. pylori IgG titer, age, year of birth, eradication history, and endoscopic findings suggesting past infection.

\section{Independent risk factors for being pseudo group A owing to past infection}

On multivariate analysis, age and year of birth were independent risk factors for being misclassified into group A despite past $H$. pylori infection (Table 5). The cutoff point for age was 50.5 years (sensitivity $72.1 \%$ and specificity 53.9\%) on ROC curve analysis $(\mathrm{p}<0.001)$. The cutoff point for year of birth was 1959.5 (sensitivity 60.6\% and specificity 61.2\%, p<0.001) for being classified into pseudo group A (Fig. 2).

\section{DISCUSSION}

In this study, 22.2\% of the group A subjects had past H. py-
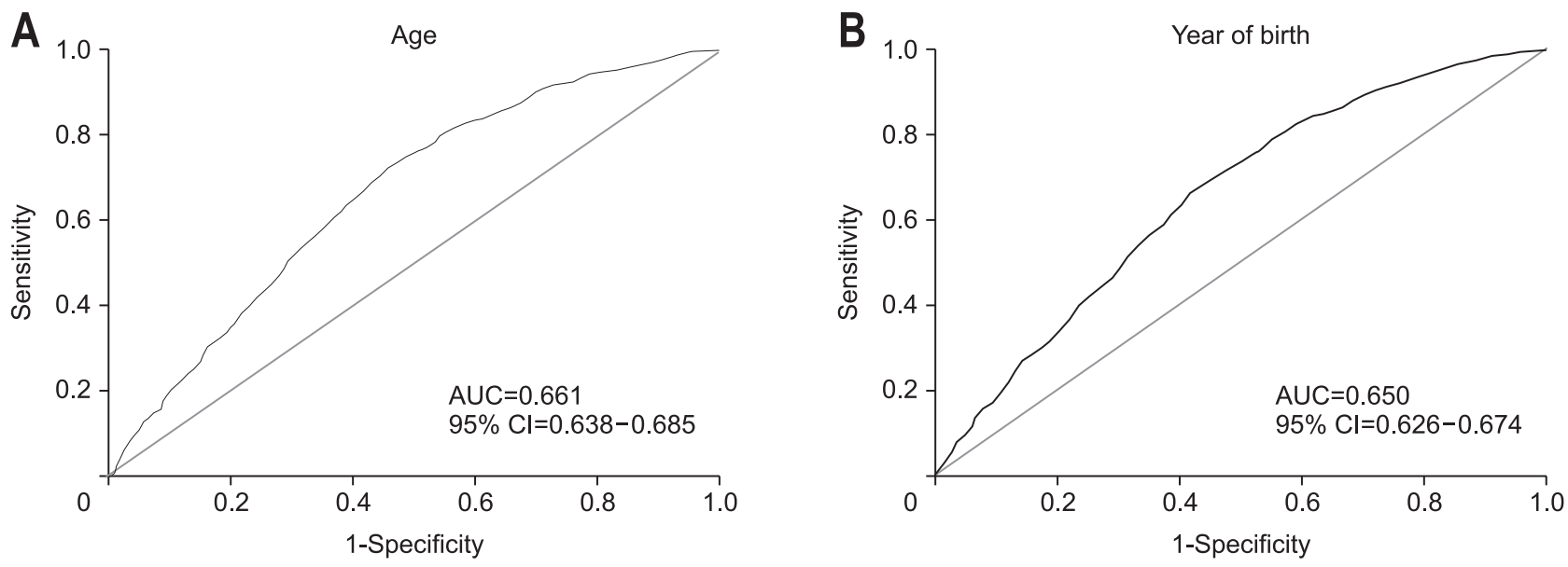

Fig. 2. Cutoff points for classification into "pseudo group A." (A, B) The cutoff points for age and year of birth were 50.5 years (sensitivity of $72.1 \%$ and specificity of 53.9\%) and 1959.5 (sensitivity of $60.6 \%$ and specificity of $61.2 \%$ ), respectively.

AUC, area under the curve; $\mathrm{CI}$, confidence interval.

Table 5. Risk Factors for Misclassification into Group A Despite Past Helicobacter pylori Infection

\begin{tabular}{|c|c|c|c|c|}
\hline \multirow{2}{*}{ Significant variable } & \multicolumn{2}{|c|}{ Univariate analysis } & \multicolumn{2}{|c|}{ Multivariate analysis } \\
\hline & OR $(95 \% \mathrm{CI})$ & $\mathrm{p}$-value & OR $(95 \%$ CI $)$ & $\mathrm{p}$-value \\
\hline Old age & $1.052(1.043-1.061)$ & $<0.001$ & $1.148(1.067-1.236)$ & $<0.001$ \\
\hline Year of birth & $0.955(0.947-0.963)$ & $<0.001$ & $1.086(1.009-1.168)$ & 0.027 \\
\hline Male sex & $1.387(1.150-1.672)$ & 0.001 & $1.301(0.932-1.815)$ & 0.122 \\
\hline Hypertension & $1.438(1.139-1.814)$ & 0.002 & $1.120(0.839-1.496)$ & 0.443 \\
\hline Diabetes mellitus & $1.514(1.074-2.133)$ & 0.018 & 1.214 (0.806-1.829) & 0.354 \\
\hline No intake of aspirin & 1 & & 1 & \\
\hline Intake of aspirin & $1.446(1.021-2.048)$ & 0.038 & $1.196(0.771-1.853)$ & 0.424 \\
\hline Never smoker & 1 & & 1 & \\
\hline Current smoker & $1.179(0.884-1.572)$ & 0.262 & $1.085(0.785-1.498)$ & 0.622 \\
\hline Past smoker & $0.701(0.516-0.954)$ & 0.024 & 0.748 (0.510-1.096) & 0.137 \\
\hline
\end{tabular}

$\mathrm{OR}$, odds ratio; $\mathrm{CI}$, confidence interval. 
lori infection, and were found to be misclassified into group A. The subjects aged over 50 years and those born before 1960 were included in the high-risk groups for being misclassified into pseudo group A, either by previous eradication or spontaneous regression of $H$. pylori. Moreover, 3.1\% of the subjects had current infection; however, being classified into pseudo group A owing to past infection did not increase the risk of current infection. Positive $H$. pylori test findings were correlated only with the PG I and PG II levels. Taken together, past infection should not be neglected in older subjects, and current infection should be ruled out in subjects with increased gastric secretory ability when using the $\mathrm{ABC}$ classification in a seroprevalent population.

Seroreversion usually occurs within a few years after $H$. pylo$r i$ eradication or spontaneous regression. ${ }^{23}$ In addition to a rapid decrease in the anti-H. pylori IgG titer, the gastric secretory ability is often recovered after $H$. pylori regression. ${ }^{24}$ Therefore, many seroreversed subjects with past $H$. pylori infection can be misclassified into group A as shown in this study. In addition to those subjects who underwent intended $H$. pylori eradication, subjects after unintended eradication or those who showed spontaneous regression also revealed normal serum PG assay findings after seroreversion. Nonetheless, the gastric cancer risk remains high in the group A subjects with past infection. ${ }^{12}$ In our study, gastric neoplasm was found in three subjects in group A (0.11\%), and two subjects showed endoscopic findings suggesting past infection. This is consistent with the findings of a recent study showing that most of the group A patients with gastric cancer had past $H$. pylori infection. ${ }^{25}$

Older age was an independent risk factor for being classified into pseudo group A, with a cutoff point of 50.5 years in this study. Furthermore, group A Koreans born before 1960 had a higher risk. Mean year of birth was most remote (1955) in subjects with spontaneous regression, and was most recent (1963) in subjects without evidence of past infection. In subjects after successful eradication, mean year of birth (1958) was closer to that of subjects with spontaneous regression than subjects without evidence of past infection. Our findings are supported by the fact that more than 40\% of group A subjects show atrophy on endoscopic findings, especially when they are older. ${ }^{26}$ Owing to these reasons, the $\mathrm{ABC}$ classification system is less useful for an older, seroprevalent population. ${ }^{27}$ Older subjects should be considered as having a high risk for gastric cancer owing to the high probability of past infection.

Another notable finding in this study is that 3.1\% of the 715 group A subjects who underwent additional $H$. pylori test showed positive findings. Along with the seroreversed pseudo group A subjects with past infection, subjects with ongoing infection should not be neglected. False seronegative findings in infected Koreans may be due to non-availability of Korean serology assays at the clinic. The only Korean immunoassay, "Genesis H. pylori ELISA" from Green Cross Medical Science is available only for research purposes, and does not show quantitative results. Therefore, imported $H$. pylori IgG assays, which exhibited a similar diagnostic accuracy with the Genedia $H$. pylori ELISA in Koreans, were used in this study. ${ }^{20}$ Nonetheless, 22 infected subjects were misclassified into the seronegative group. Because these subjects showed high mean PG I (75.3 ng/mL) and PG II (16.4 ng/mL) levels, this could be improved by adding other $H$. pylori tests to the group A subjects with increased PG levels.

The pseudo group A subjects with eradication history showed a higher serum anti-H. pylori IgG titer than those with abnormal endoscopic findings suggesting past infection. Nonetheless, classification into pseudo group A and its correlated variables (i.e., old age, male sex, and earlier year of birth) did not increase the risk of current infection. The lack of a correlation between the increased serology titer and current infection supports the finding that the $\operatorname{IgG}$ titers decrease slowly after eradication. ${ }^{14}$ Despite such limitations, the $\mathrm{ABC}$ classification using the PG assay is still useful in Koreans with eradication history, because atrophy and metaplastic gastritis can be improved after eradication. ${ }^{28}$ Another Korean study showed that the PG I/II ratio of subjects becomes similar to that of normal controls after 5 years of eradication. ${ }^{29}$ The gastric biomarker levels in subjects with eradication history seem to recover to the level in noninfected subjects after 3 years, although a longer duration is needed in subjects with severe corpus atrophy. ${ }^{30,31}$

There are limitations in our study. First, eradication history was based on the answers to questionnaires. There might have been a recall bias; however, the answers are usually reliable because it is not easy to forget 7 to 14 days of antibiotic intake for $H$. pylori eradication by intention. Moreover, in a seroprevalent population, the risk of providing imprecise information on past eradication might be lower than the risk of unintended eradication or spontaneous regression. Therefore, we hypothesized that the proportion of misclassified pseudo group A subjects owing to recall bias is small, and would not affect the main findings of this study. Second, additional $H$. pylori tests were performed only in 715 subjects. Nevertheless, we could verify that positive H. pylori test findings are correlated with the PG I and PG II levels, and not with the pseudo group A-related variables. This further suggests that current infection is uncommon even in pseudo group A subjects with a high serology titer.

In conclusion, 22.2\% of the group A subjects were misclassified despite the gastric cancer risk, and the efficacy of the $\mathrm{ABC}$ classification method is limited owing to these pseudo group A seroreversed subjects. Along with pseudo group A subjects with past infection, those with current $H$. pylori infection can be misclassified into group A. Ongoing $H$. pylori infection should be considered in subjects with increased PG I and PG II levels, regardless of their age, serology titer, eradication history, and endoscopic findings. To discriminate seroreversed subjects with a risk of gastric cancer, past infection should be assessed in sub- 
jects aged over 50 years, especially in those born before 1960 . Furthermore, current infection should be ruled out in subjects with increased gastric secretory ability.

\section{CONFLICTS OF INTEREST}

No potential conflict of interest relevant to this article was reported.

\section{ACKNOWLEDGEMENTS}

This work was supported by Konkuk University and National Research Foundation (2016R1D1A1B02008937).

\section{AUTHOR CONTRIBUTIONS}

Drafting of the manuscript: H.K., S.Y.L. Study concept and design: S.Y.L. Data acquisition, data analysis and interpretation, and statistical analysis: H.K., J.H.K., S.P.L. Administrative, technical support, and study supervision: J.H.K., I.K.S., H.S.P., C.S.S.

\section{ORCID}

Sun-Young Lee https://orcid.org/0000-0003-4146-6686

\section{REFERENCES}

1. Plummer M, Franceschi S, Vignat J, Forman D, de Martel C. Global burden of gastric cancer attributable to Helicobacter pylori. Int J Cancer 2015;136:487-490.

2. Hooi JKY, Lai WY, Ng WK, et al. Global prevalence of helicobacter pylori infection: systematic review and meta-analysis. Gastroenterology 2017;153:420-429.

3. Peleteiro B, Bastos A, Ferro A, Lunet N. Prevalence of Helicobacter pylori infection worldwide: a systematic review of studies with national coverage. Dig Dis Sci 2014;59:1698-1709.

4. Carneiro F. Stomach cancer. In: Steward B, Wild CP, eds. World cancer report. Lyon: International Agency for Research on Cancer, 2014:383-391.

5. Shin A, Park S, Shin HR, et al. Population attributable fraction of infection-related cancers in Korea. Ann Oncol 2011;22:14351442.

6. Lee JH, Choi KD, Jung HY, et al. Seroprevalence of Helicobacter pylori in Korea: a multicenter, nationwide study conducted in 2015 and 2016. Helicobacter 2018;23:e12463.

7. Miki K, Fujishiro M, Kodashima S, Yahagi N. Long-term results of gastric cancer screening using the serum pepsinogen test method among an asymptomatic middle-aged Japanese population. Dig Endosc 2009;21:78-81.

8. Yamaguchi Y, Nagata Y, Hiratsuka R, et al. Gastric cancer screening by combined assay for serum anti-Helicobacter pylori IgG antibody and serum pepsinogen levels: the ABC Method. Digestion
2016;93:13-18.

9. Miki K. Gastric cancer screening by combined assay for serum anti-Helicobacter pylori IgG antibody and serum pepsinogen levels: “ABC method”. Proc Jpn Acad Ser B Phys Biol Sci 2011;87:405414.

10. Choi HS, Lee SY, Kim JH, et al. Combining the serum pepsinogen level and Helicobacter pylori antibody test for predicting the histology of gastric neoplasm. J Dig Dis 2014;15:293-298.

11. Watabe H, Mitsushima T, Yamaji Y, et al. Predicting the development of gastric cancer from combining Helicobacter pylori antibodies and serum pepsinogen status: a prospective endoscopic cohort study. Gut 2005;54:764-768.

12. Kiso M, Yoshihara M, Ito M, et al. Characteristics of gastric cancer in negative test of serum anti-Helicobacter pylori antibody and pepsinogen test: a multicenter study. Gastric Cancer 2017;20:764771.

13. Kishikawa H, Kimura K, Ito A, et al. Cutoff pepsinogen level for predicting unintendedly eradicated cases of Helicobacter pylori infection in subjects with seemingly normal pepsinogen levels. Digestion 2017;95:229-236.

14. Chinda D, Shimoyama T, Mikami T, et al. Serum pepsinogen levels indicate the requirement of upper gastrointestinal endoscopy among group A subjects of $\mathrm{ABC}$ classification: a multicenter study. J Gastroenterol 2018;53:924-931

15. Boda T, Ito M, Yoshihara M, et al. Advanced method for evaluation of gastric cancer risk by serum markers: determination of true low-risk subjects for gastric neoplasm. Helicobacter 2014;19:1-8.

16. Maehata Y, Nakamura S, Esaki M, et al. Characteristics of primary and metachronous gastric cancers discovered after Helicobacter pylori eradication: a multicenter propensity score-matched study. Gut Liver 2017;11:628-634.

17. Lee SP, Lee SY, Kim JH, Sung IK, Park HS, Shim CS. Link between serum pepsinogen concentrations and upper gastrointestinal endoscopic findings. J Korean Med Sci 2017;32:796-802.

18. Kimura K, Takemoto T. An endoscopic recognition of the atrophic border and its significance in chronic gastritis. Endoscopy 1969;1:87-97.

19. Sugimoto M, Ban H, Ichikawa H, et al. Efficacy of the Kyoto classification of gastritis in identifying patients at high risk for gastric cancer. Intern Med 2017;56:579-586.

20. Lee SY, Moon HW, Hur M, Yun YM. Validation of western Helicobacter pylori IgG antibody assays in Korean adults. J Med Microbiol 2015;64:513-518.

21. Kim JH, Lee SY, Lee SP, et al. The histologic detection of Helicobacter pylori in seropositive subjects is affected by pathology and secretory ability of the stomach. Helicobacter 2018;23:e12480.

22. Lee SY. Endoscopic gastritis, serum pepsinogen assay, and Helicobacter pylori infection. Korean J Intern Med 2016;31:835-844.

23. Koizumi W, Tanabe S, Imaizumi H, et al. Effect of anti-Helicobacter pylori IgG antibody titer following eradication of Helicobacter pylori infection. Hepatogastroenterology 2003;50:293-296.

24. Ohkusa T, Miwa H, Nomura T, et al. Improvement in serum pep- 
sinogens and gastrin in long-term monitoring after eradication of Helicobacter pylori: comparison with $\mathrm{H}$. pylori-negative patients. Aliment Pharmacol Ther 2004;20:25-32.

25. Miura K, Okada H, Kouno Y, et al. Actual status of involvement of Helicobacter pylori infection that developed gastric cancer from group $\mathrm{A}$ of $\mathrm{ABC}$ (D) Stratification - study of early gastric cancer cases that underwent endoscopic submucosal dissection. Digestion 2016;94:17-23.

26. Kotachi T, Ito M, Yoshihara M, et al. Serological evaluation of gastric cancer risk based on pepsinogen and Helicobacter pylori antibody: relationship to endoscopic findings. Digestion 2017;95:314318.

27. Shimoyama T, Aoki M, Sasaki Y, Matsuzaka M, Nakaji S, Fukuda $\mathrm{S}$. ABC screening for gastric cancer is not applicable in a Japanese population with high prevalence of atrophic gastritis. Gastric Can- cer 2012;15:331-334

28. Kang JM, Kim N, Shin CM, et al. Predictive factors for improvement of atrophic gastritis and intestinal metaplasia after Helicobacter pylori eradication: a three-year follow-up study in Korea. Helicobacter 2012;17:86-95.

29. Nam SY, Jeon SW, Lee HS, Kwon YH, Park H, Choi JW. Longterm follow-up of pepsinogen I/II ratio after eradication of Helicobacter pylori in patients who underwent endoscopic mucosal resection for gastric cancer. Dig Liver Dis 2017;49:500-506.

30. Daugule I, Ruskule A, Moisejevs G, et al. Long-term dynamics of gastric biomarkers after eradication of Helicobacter pylori infection. Eur J Gastroenterol Hepatol 2015;27:501-505.

31. Vannella L, Lahner E, Bordi C, et al. Reversal of atrophic body gastritis after H. pylori eradication at long-term follow-up. Dig Liver Dis 2011;43:295-299. 